\title{
Brueckner correlations following a boson mapping of the two-color delta model
}

\author{
M. V. Stoitsov, ${ }^{1,2}$ S. Pittel, ${ }^{2}$ and J. Dukelsky ${ }^{3}$ \\ ${ }^{1}$ Institute of Nuclear Research and Nuclear Energy, Bulgarian Academy of Sciences, Sofia-1784, Bulgaria \\ ${ }^{2}$ Bartol Research Institute, University of Delaware, Newark, Delaware 19716 \\ ${ }^{3}$ Instituto de Estructura de la Materia, Consejo Superior de Investigaciones Cientificas, Serrano 123, 28006 Madrid, Spain
}

(Received 19 December 1995)

\begin{abstract}
We study the application of boson mapping methods to a two-color delta model of interacting quarks, with the purpose of extracting information of relevance to quark-model descriptions of nuclei. In an earlier treatment, the boson Hamiltonian resulting from the mapping was approximately diagonalized at the level of Hartree-Bose approximation. The results differed from those of an exact solution of the model at first order in the density. Furthermore, the results were worse than those arising from a BCS treatment of pair correlations at the quark level. Here we extend the analysis to a Brueckner treatment, so as to properly take into account the short-range repulsion between bosons originating from the quark Pauli principle. The resulting energy per quark reproduces the exact results through first order in the density and is significantly better than the BCS results. At higher densities, deviations appear, reflecting the need for a full cluster expansion in the treatment of short-range correlation effects. The relevance of these results to more realistic three-color quark models of nuclei is discussed. [S0556-2813(96)03606-0]
\end{abstract}

PACS number(s): 21.60.Gx, 12.39.Jh, 21.30.Fe, 24.85.+p

\section{INTRODUCTION}

Traditionally, the nucleus is treated as a system of interacting nucleons. This approach is founded on the assumption that the QCD interactions that build the nucleon decouple from the residual interactions between nucleons responsible for nuclear structure. Recent experiments suggest that at some level this assumption breaks down. The EMC data, for example, suggest that the internal structure of the nucleon changes in the presence of a nuclear medium. As such, there is currently great interest in trying to develop a theory of nuclear structure directly from QCD, thereby taking into account those effects that arise from medium corrections to the structure of the nucleon.

Modifications to the structure of the nucleon are a consequence of quark exchange. Some of these effects are naturally incorporated in the traditional approach, whereby the nucleon-nucleon interaction is first derived from QCD (or some approximation to it) and then used in subsequent nuclear structure analysis. Such an approach omits quark exchange effects involving several nucleons, however. It is generally thought that such effects should not be important at normal (relatively dilute) nuclear densities, except perhaps in dynamical processes involving large momentum transfers. However, in the absence of a fully consistent analysis that incorporates on the same footing the effects of QCD in building the nucleon and also in nuclear structure, this remains unproven. From our perspective, it is important, therefore, to build a theory that consistently incorporates these various aspects of QCD. A theory of this type, able to reproduce traditional nuclear properties, could then help isolate where to look for explicit quark effects in nuclear structure.

While such a consistent theory should ideally start from QCD, this is not currently practical. What is required instead is an effective theory that builds in (at least approximately) the important properties of QCD, while still remaining tractable. Constituent quark models seem to satisfy these criteria.
Though not derived directly from QCD, these models incorporate at some level several of its key ingredients, including, for example, approximate confinement [1] and approximate chiral invariance [2]. Furthermore, they have been shown to reproduce with impressive success the properties of nuclear systems with very few particles. What is much less clear, however, is how to apply these models to many-body systems. Since a system of $3 A$ quarks clusters into $A$ triplets (nucleons) at normal nuclear densities, a necessary ingredient is a method of handling strong three-quark correlations in a many-quark environment, a scenario that cannot be handled with traditional many-body approaches.

Recently, a method was proposed [3] for treating multinucleon systems in a constituent quark framework using mapping techniques. The earliest tests of these methods were to models that did not admit spatial three-quark correlations [3,4], a key ingredient of any realistic quark description of nuclei. For that reason, the method was subsequently applied to a series of models due to Koltun and collaborators $[8,9]$, in which quarks (with color) move nonrelativistically in one dimension (1D) and interact through a residual attractive delta-function potential. Despite severe limitations of the model-one-dimensional motion, schematic interactions, nonrelativistic dynamics-there are several features of this model that nevertheless make it attractive as a testing ground of quark mapping methods. At low densities, the model exhibits spatial correlations between clusters of quarks. Equally important, the model can be exactly solved as a function of the density of the system using the Bethe ansatz.

Models of this type have been developed both for two and three colors. While the three-color version certainly has a more obvious (albeit still schematic) connection to quark dynamics in nuclei, the two-color model is also of interest to study in view of its greater simplicity.

An analysis of the two-color model was reported in Ref. [5]. Following a boson mapping [7] of the model, the resulting Hamiltonian was treated in the Hartree-Bose approxima- 
tion. The resulting energy per quark was found to differ from the exact results at first (linear) order in the density. Furthermore, the results were somewhat worse than achieved by a simple BCS treatment of the same model, even though that analysis likewise was unable to reproduce the linear term in density correctly. It should be noted here that for this model effects of the interaction between clusters can first contribute at linear order in the density.

A corresponding analysis of the more physically relevant three-color model was reported in [6]. There, a baryon mapping of the model was carried out and the resulting Hamiltonian was then treated in the Hartree-Fock approximation. Very similar conclusions to those of the two-color analysis were obtained. The mapping could be implemented, but a subsequent mean-field treatment could not adequatly reproduce the exact dynamics. There, the resulting energy per quark differed from the exact results at third order in the density. But this too is the lowest order in which the interactions between clusters contribute; the fermionic nature of this model guarantees that the cluster-cluster interaction does not contribute to the energy in either first or second order.

Clearly, a many-body method of cluster phenomena that is unable to treat correctly the interactions between clusters, even to lowest order, cannot be used with any degree of confidence. Mapping techniques, coupled with a pure meanfield analysis, are unable to accomplish this for either of the two cases studied.

Mapping methods, however, are not limited to a pure mean-field analysis. As discussed in [5,6], the mapping also provides the interaction between clusters. This has two important consequences. On the one hand, it provides the information needed to isolate the physics that was "missing" from the pure mean-field treatment. Equally important, once this physics has been appropriately identified, it provides a framework to incorporate these additional correlations.

In our analysis of both the two- and three-color models, the cluster-cluster interaction that emerged from the mapping was found to be strongly repulsive at short distances. As a consequence, we suggested, both in $[5,6]$, that Brueckner theory [10] might be the key missing ingredient required to improve the usefulness of mapping methods in the presence of spatial clustering. The situation is analogous to that of traditional nuclear physics. As is well known, the bare nucleon-nucleon interaction contains a strong short-range repulsive component, originating in quark exchange (though often simulated by $\omega$ exchange), and this precludes a direct mean-field treatment.

The purpose of the present work is to assess the above conjecture that including Brueckner correlations significantly improves the ability of mapping techniques to describe the cluster dynamics of quark models. Since the three-color model is so much more difficult to treat than the two-color model, though certainly more relevant to quarks, we have chosen to address this issue in the context of the simpler two-color model. Thus, we report here a Brueckner treatment of the boson Hamiltonian that results from a boson mapping of the two-color model.

The key result of our analysis is that by including Brueckner correlations we are able to achieve perfect agreement with the exact linear term in the energy per quark. This suggests that the same conclusions would most likely be ob- tained for the more realistic three-color model. Namely, if we were to include short-range correlation effects in a Brueckner-Hartree-Fock analysis, we should be able reproduce the exact results of that model through third order in the density, i.e., through the lowest order in which the interactions between the relevant three-particle clusters (nucleons) contribute.

The structure of the paper is as follows: In Sec. II, we briefly summarize some essential features of the two-color delta model, including its treatment using the BCS method. In Sec. III, we review our earlier application of bosonmapping techniques to the model. In Sec. IV, we describe our Brueckner analysis and report the results. Sec. V contains some concluding remarks, including comments on the relevance of the work to the three-color model and to more realistic quark models of nuclei.

\section{THE TWO-COLOR DELTA MODEL}

\section{A. The model}

In this model, a system of $N$ nonrelativistic quarks with color $c$ (which is allowed to take the two possible values $\pm 1 / 2$ ) move in a one-dimensional (1D) box of size $L$ subject to an attractive delta-function interaction. Letting $q_{k c}^{\dagger}\left(q_{k c}\right)$ denote the creation (annihilation) operator for a quark with momentum $k$ and color $c$, we can express the model Hamiltonian as $(\hbar=1)$

$$
H=\sum_{k c} \varepsilon_{k} q_{k c}^{\dagger} q_{k c}-\frac{G}{2} \sum_{i j k l, c} q_{i c}^{\dagger} q_{j-c}^{\dagger} q_{l-c} q_{k c} \delta_{i+j, k+l},
$$

where $G$ is the strength of the interaction and $\varepsilon_{k}=k^{2} / 2 m$. The infinite-matter limit, $N \rightarrow \infty, L \rightarrow \infty \quad(\rho=N / L$ finite), is then obtained by replacing

$$
\sum_{k} \rightarrow \frac{L}{2 \pi} \int d k, \quad \delta_{i j} \rightarrow \frac{2 \pi}{L} \delta(i-j), \quad G \rightarrow g / L .
$$

\section{B. Exact solution at low densities}

In the infinite-matter limit, the ground state energy per particle can be obtained exactly by solving the following set of integral equations [8]:

$$
\begin{gathered}
\rho=2 \int_{-K}^{K} F(k, K) d k, \\
\frac{E}{N}=-\frac{m g^{2}}{8}+\frac{2}{\rho} \int_{-K}^{K} \varepsilon_{k} F(k, K) d k,
\end{gathered}
$$

where the density function for doublet clusters $F(k, K)$ satisfies the relation

$$
\pi F(k, K)=1-\int_{-K}^{K}\left[\frac{m g F(\omega, K)}{(m g)^{2}+(k-\omega)^{2}}\right] d \omega .
$$

These equations scale with the dimensionless parameter $\widetilde{\rho}=\rho /(m g)$. From this, it is straightforward to obtain the energy per particle numerically at any density. Here, we present the analytic results of an expansion in powers of $\tilde{\rho}$ through third order: 


$$
\frac{E}{N} \frac{1}{m g^{2}}=-\frac{1}{8}+\frac{\pi^{2} \widetilde{\rho}^{2}}{96}+\frac{\pi^{2} \widetilde{\rho}^{3}}{96}+O\left(\widetilde{\rho}^{4}\right) .
$$

Note that no linear term in $\widetilde{\rho}$ appears in Eq. (6).

\section{BCS treatment}

A BCS treatment of the model leads to the familiar number and gap equations [8]

$$
\rho=\frac{1}{L} \sum_{k}\left[1-\frac{\bar{\varepsilon}_{k}^{2}}{\sqrt{\left(\bar{\varepsilon}_{k}^{2}+\Delta^{2}\right)}}\right], \quad \frac{2}{G}=\sum_{k} \frac{1}{\sqrt{\left(\bar{\varepsilon}_{k}^{2}+\Delta^{2}\right)}},
$$

where $\lambda$ is the chemical potential, $\bar{\varepsilon}_{k}=\varepsilon_{k}-\lambda-G N / 2$, $\Delta=G \Sigma_{k} u_{k} v_{k}$ is the pairing gap, and $\rho=(2 / L) \Sigma_{k} v_{k}^{2}$ is the density of the system. Equations (7) are solved for the chemical potential and the pairing gap. The resulting BCS energy is then given by

$$
E_{\mathrm{BCS}}=2 \sum_{k} \varepsilon_{k} v_{k}^{2}-G \sum_{k k^{\prime}}\left(u_{k} v_{k} u_{k^{\prime}} v_{k^{\prime}}+v_{k}^{2} v_{k^{\prime}}^{2}\right),
$$

where

$$
v_{k}^{2}=\frac{1}{2}\left[1-\frac{\bar{\varepsilon}_{k}^{2}}{\sqrt{\left(\overline{\varepsilon_{k}^{2}}+\Delta^{2}\right)}}\right], \quad u_{k}^{2}=\frac{1}{2}\left[1+\frac{\bar{\varepsilon}_{k}^{2}}{\sqrt{\left(\bar{\varepsilon}_{k}^{2}+\Delta^{2}\right)}}\right] .
$$

Solutions to the BCS equations in powers of $\widetilde{\rho}$ are given in [4]. The energy per quark through second order in $\widetilde{\rho}$ that results is

$$
\frac{E_{\mathrm{BCS}}}{N} \frac{1}{m g^{2}}=-\frac{1}{8}+\frac{\tilde{\rho}}{8}+\frac{\tilde{\rho}^{2}}{16}+O\left(\tilde{\rho}^{3}\right) .
$$

The BCS approximation reproduces correctly the zerothorder term but yields a first-order contribution not present in the exact results. Nevertheless, it is important to note that, to date, this is the best approximate treatment of the model that has been reported.

\section{DYSON BOSON MAPPING APPLIED TO THE MODEL}

\section{A. Boson mapping}

The Dyson mapping [7] is based on the requirement that the commutation algebra of bilinear quark operators be preserved by their mapping onto boson operators. A key feature of this mapping is that it is finite but non-Hermitean.

Application of the Dyson method to the model of interest involves mapping a colorless quark pair $\Sigma_{c}(-)^{1 / 2-c} q_{c k_{1}}^{\dagger} q_{-c k_{2}}^{\dagger}$ onto a colorless boson $\Gamma_{k K}^{\dagger}$. Here $k=(1 / 2)\left(k_{1}-k_{2}\right)$ is the relative momentum of the quark pair and $K=k_{1}+k_{2}$ is its total momentum. The colorless creation operator $\Gamma_{k K}^{\dagger}$ and the corresponding colorless annihilation operator $\Gamma_{k K}$ satisfy the commutation relation

$$
\left[\Gamma_{k K}, \Gamma_{k^{\prime} K^{\prime}}^{\dagger}\right]=\delta_{K K^{\prime}}\left(\delta_{k k^{\prime}}+\delta_{k-k^{\prime}}\right) .
$$

For our purposes, the key result is that the quark Hamiltonian (1) is mapped onto a boson Hamiltonian

$$
H_{B}=H_{0}+V_{B} \text {, }
$$

where

$$
H_{0}=\sum_{k k^{\prime} K}\left[\left(\frac{k^{2}}{2 m}+\frac{K^{2}}{8 m}\right) \delta_{k k^{\prime}}-\frac{g}{2 L}\right] \Gamma_{k K}^{\dagger} \Gamma_{k^{\prime} K},
$$

and

$$
V_{B}=\frac{g}{4 L} \sum_{\substack{k k^{\prime} q \\ K K^{\prime}}} \Gamma_{\frac{q}{2}-k^{\prime}-\frac{K^{\prime}-K}{2}, q+K}^{\dagger} \Gamma_{k^{\prime}-\frac{q}{2}, K^{\prime}-q}^{\dagger} \Gamma_{k^{\prime} K^{\prime}} \Gamma_{k K}
$$

To apply known many-body techniques to the Hamiltonian (12)-(14), we introduce a (nonunitary) collective transformation [11]

$$
\Lambda_{p K}^{\dagger}=\sum_{k} X_{k p}^{K} \Gamma_{k K}^{\dagger}, \quad \lambda_{p K}=\sum_{k} Y_{k p}^{K} \Gamma_{k K},
$$

where

$$
\left[\lambda_{p K}, \Lambda_{p^{\prime} K^{\prime}}^{\dagger}\right]=\delta_{p p^{\prime}} \delta_{K K^{\prime}},
$$

and the coefficients of the collective transformation satisfy

$$
\begin{gathered}
\sum_{k} X_{k p}^{K} Y_{k p^{\prime}}^{K^{\prime}}=\frac{1}{2} \delta_{p p^{\prime}} \delta_{K K^{\prime}}, \\
\sum_{p} X_{k^{\prime} p}^{K} Y_{k p}^{K^{\prime}}=\frac{1}{4}\left(\delta_{k k^{\prime}}+\delta_{k-k^{\prime}}\right) \delta_{K K^{\prime}} .
\end{gathered}
$$

The collective transformation (15) can be readily inverted, giving

$$
\Gamma_{k K}^{\dagger}=2 \sum_{p} Y_{k p}^{K} \Lambda_{p K}^{\dagger}, \quad \Gamma_{k K}=2 \sum_{p} X_{k p}^{K} \lambda_{p K} .
$$

To obtain an appropriate collective Hamiltonian to use in describing the ground state of the system, we truncate to the lowest collective $(p=1)$ boson for each $K$ value. To simplify the notation, we suppress the label $p$ in all subsequent expressions; $\quad$ viz. $\quad\left(\Lambda_{p=1 K}^{\dagger}, \lambda_{p=1 K}, X_{k p=1}^{K}, Y_{k p=1}^{K}\right) \rightarrow$ $\left(\Lambda_{K}^{\dagger}, \lambda_{K}, X_{k}^{K}, Y_{k}^{K}\right)$.

The resulting collective Hamiltonian can then be written as

$$
H_{B}=\sum_{K} \epsilon_{K} \Lambda_{K}^{\dagger} \lambda_{K}+\sum_{q, \Delta, K} f(q, \Delta, K) \Lambda_{q+K}^{\dagger} \Lambda_{K+\Delta-q}^{\dagger} \lambda_{K+\Delta} \lambda_{K},
$$

where

$$
\epsilon_{K}=\sum_{k^{\prime}}\left(\frac{2 k^{\prime 2}}{m}+\frac{K^{2}}{2 m}\right) X_{k^{\prime}}^{K} Y_{k^{\prime}}^{K}-\frac{2 g}{L} \sum_{k^{\prime} k^{\prime \prime}} X_{k^{\prime}}^{K} Y_{k^{\prime \prime}}^{K},
$$

and

$$
f(q, \Delta, K)=\frac{4 g}{L} \sum_{k^{\prime} k^{\prime \prime}} Y_{k^{\prime}-(\Delta-q) / 2}^{K+q} Y_{k^{\prime}-(q / 2)}^{K+\Delta-q} X_{k^{\prime}}^{K+\Delta} X_{k^{\prime \prime}}^{K} .
$$




\section{B. Hartree-Bose approximation}

We now summarize the key features of our earlier application of the Hartree-Bose (HB) approximation to the above boson system. The Hartree Bose approximation is a variational approximation based on a boson condensate trial state

$$
\left|\Phi_{0}\right\rangle=\frac{1}{\sqrt{N_{B} !}} \Lambda_{0}^{\dagger N_{B}}|0\rangle, \quad\left\langle\Psi_{0}\right|=\frac{1}{\sqrt{N_{B} !}}\langle 0| \lambda_{0}{ }^{N_{B}},
$$

where $N_{B}=N / 2$. The variational condition is to minimize

$$
E_{\mathrm{HB}}(\lambda)=\left\langle\Psi_{0}\left|H_{B}-\lambda \hat{N}_{B}\right| \Phi_{0}\right\rangle
$$

subject to the normalization constraint $\Sigma_{k} X_{k}^{0} Y_{k}^{0}=1 / 2$.

The boson condensate (22) only involves collective $K=0$ bosons, since they are the lowest in energy. The HB condition, thus, provides a variational prescription for generating the structure coefficients of the $K=0$ bosons only, viz. $X_{k}^{0}$ and $Y_{k}^{0}$. For simplicity, we denote these collective structure coefficients by $x_{k}$ and $y_{k}$, respectively. The relevant energy functional to be minimized, subject to the normalization constraint, is

$$
\begin{aligned}
E_{\mathrm{HB}}(\lambda)= & N \sum_{k}\left(\frac{k^{2}}{m}-\lambda\right) x_{k} y_{k}-\frac{g N}{L} \sum_{k k^{\prime}} x_{k} y_{k} \\
& +\frac{g N^{2}}{L} \sum_{k k^{\prime}} y_{k^{\prime}}^{2} x_{k^{\prime}} x_{k} .
\end{aligned}
$$

Note that in Eq. (24), we have thrown away all terms down by $1 / N$ relative to the terms retained, as is appropriate in the infinite-matter limit.

The solution of the HB variational equations was discussed in [5]. An important feature is that the structure functions can be directly related to the $u_{k}$ and $v_{k}$ coefficients of the BCS approximation, through the introduction of a physical condition. In particular,

$$
x_{k}=\sqrt{\frac{1}{N}} v_{k} u_{k}, \quad y_{k}=\sqrt{\frac{1}{N}} \frac{v_{k}}{u_{k}} .
$$

As a consequence, the HB structure functions can be obtained from the same gap and number equations that arise in the BCS approximation (7). For our purposes, the principal result that emerges is that the energy per quark of the HB approximation through first order in $\tilde{\rho}$ is

$$
\frac{E_{\mathrm{HB}}}{N} \frac{1}{m g^{2}}=-\frac{1}{8}+\frac{3 \tilde{\rho}}{8}+O\left(\tilde{\rho}^{2}\right) .
$$

Thus, like BCS approximation, the HB analysis also produces a linear term in the energy per particle. However, the linear term that arises is three times larger than in BCS, so that the agreement with the exact results is even worse.

At first glance, the results of our HB analysis might seem discouraging, since they suggest that mapping methods cannot incorporate correlations as well as simpler quasiparticle methods carried out on the original quark model. Such a conclusion is premature, however. As we now discuss, mapping methods provide a natural framework for systematically going beyond the mean field and thus improving on the HB results.

The key point is that the mapping provides not only the Hamiltonian involving $K=0$ bosons (those that enter in HB approximation) but also [see Eq. (21)] those that connect the HB condensate to "excited" $K \neq 0$ bosons.

The collective boson Hamiltonian given in Eq. (21) depends on the structure amplitudes for the dominant collective bosons with all $K$ values. It is only the structure of the collective $K=0$ bosons, however, that is provided by the HB analysis. To obtain the collective Hamiltonian required for analysis beyond the HB approximation, we also need the structure of the collective $K \neq 0$ bosons. In the numerical calculations to follow, we consider two approaches. In the first, we assume that the internal structure of the collective $K \neq 0$ bosons is the same as for the $K=0$ bosons that derive from the HB approximation. Such an assumption is precisely true at zero-density, from translational invariance arguments, but not at finite density. Thus, we also discuss in the next subsection an alternative means of generating the structure of the collective $K \neq 0$ bosons, using the Tamm-Dancoff (TD) approximation.

\section{Tamm-Dancoff approximation}

At very low densities, a meaningful prescription for the internal structure of the $K \neq 0$ collective bosons is provided by the TD approximation. Here, one assumes for each $K$ $\neq 0$ value a variational trial state of the form

$\left|\phi_{K}\right\rangle=\frac{1}{\sqrt{N_{B}}} \Lambda_{K}^{\dagger} \lambda_{0}\left|\Phi_{0}\right\rangle, \quad\left\langle\psi_{K}\right|=\frac{1}{\sqrt{N_{B}}}\left\langle\Psi_{0}\right| \Lambda_{0}^{\dagger} \lambda_{K}$,

where $\left|\Phi_{0}\right\rangle$ and $\left\langle\Psi_{0}\right|$ are the Hartree-Bose ground state wave functions (22).

The expectation value of the Hamiltonian (19) taken between the states (27) defines the variational energy functional

$$
\begin{aligned}
E_{T D}^{K}\left(\mu_{K}\right)=\left\langle\psi_{K}\left|H_{B}-\mu_{K} \hat{N}_{B}\right| \phi_{K}\right\rangle-E_{\mathrm{HB}}\left(\mu_{K}\right)= & 2 \sum_{k}\left[\left(\frac{k^{2}}{m}+\frac{K^{2}}{4 m}\right)-\mu_{K}\right] X_{k}^{K} Y_{k}^{K}-\frac{2 g}{L} \sum_{k k^{\prime}} X_{k}^{K} Y_{k^{\prime}}^{K}+\frac{2 g N}{L} \sum_{k k^{\prime}} x_{k^{\prime}} y_{k^{\prime}} X_{k}^{K}\left(Y_{k^{\prime}-K / 2}^{K}\right. \\
& \left.+Y_{k^{\prime}+K / 2}^{K}\right)+\frac{2 g N}{L} \sum_{k k^{\prime}} X_{k^{\prime}}^{K} Y_{k^{\prime}}^{K} x_{k}\left(y_{k^{\prime}-K / 2}+y_{k^{\prime}+K / 2}\right)
\end{aligned}
$$


The TD approximation involves minimizing this functional with respect to the variational structure amplitudes $X_{k}^{K}$ or $Y_{k}^{K}$, subject to the normalization constraint $\Sigma_{k} X_{k}^{K} Y_{k}^{K}=1 / 2$ [see Eq. (17)]. As in the Hartree-Bose treatment of [4], we impose a physical condition that relates the $X_{k}^{K}$ and $Y_{k}^{K}$ amplitudes. Here, the appropriate condition is

$$
X_{k}^{K}=Y_{k}^{K}\left[1-N\left(x_{K-k / 2} y_{K-k / 2}+x_{K+k / 2} y_{K+k / 2}\right)\right] .
$$

Performing the variation of the functional (28) and using the normalization constraint and physical condition leads to the following solutions for the collective structure amplitudes of interest:

$$
\begin{aligned}
& Y_{k}^{K}=\frac{\Delta_{K}}{k^{2} / m+K^{2} / 4 m-\mu_{K}+\sqrt{N} \Delta\left[y_{k-K / 2}+y_{k+K / 2}\right]}, \\
& X_{k}^{K}=\frac{\Delta_{K}\left[1-N\left(x_{k-K / 2} y_{k-K / 2}+x_{k+K / 2} y_{k+K / 2}\right)\right]}{k^{2} / m+K^{2} / 4 m-\mu_{K}+\sqrt{N} \Delta\left[y_{k-K / 2}+y_{k+K / 2}\right]} .
\end{aligned}
$$

Here, $x_{k}$ and $y_{k}$ are the $K=0$ structure amplitudes derived in the HB approximation in terms of the BCS gap $\Delta$ and chemical potential $\lambda$. Also, $\mu_{K}$ and $\Delta_{K}$ satisfy Tamm-Dancoff "number" and "gap" equations

$$
1=\frac{g}{L} \sum_{k} \frac{1-N\left(x_{k-K / 2} y_{k-K / 2}+x_{k+K / 2} y_{k+K / 2}\right)}{k^{2} / m+K^{2} / 4 m-\mu_{K}+\sqrt{N} \Delta\left[y_{k-K / 2}+y_{k+K / 2}\right]}
$$

and

$$
\frac{1}{2 \Delta_{K}^{2}}=\sum_{k} \frac{1-N\left(x_{k-K / 2} y_{k-K / 2}+x_{k+K / 2} y_{k+K / 2}\right)}{\left(k^{2} / m+K^{2} / 4 m-\mu_{K}+\sqrt{N} \Delta\left[y_{k-K / 2}+y_{k+K / 2}\right]\right)^{2}} .
$$

The system of equations (30)-(32) can be numerically solved, thereby giving a variational prescription for the $Y_{k}^{K}$ and $X_{k}^{K}$ amplitudes appropriate in the limit of low densities.

\section{The collective boson Hamiltonian}

Once the structure coefficients have been obtained, we can determine the collective boson Hamiltonian from Eq. (21) and use it in many-body treatments that include correlations beyond those of the Hartree-Bose approximation.

It is useful, however, to first make some qualitative remarks about this collective Hamiltonian. As we have seen, the energy per quark derived in the HB approximation differs from the exact results already at first order in the density. To obtain the energy to this order, we only require information on the collective boson wave functions at zeroth order in $\widetilde{\rho}$, i.e., at $\widetilde{\rho}=0$. At zero-density, the structure coefficients of all collective bosons are the same and given by [5]

$$
Y_{k}^{K}=X_{k}^{K}=\sqrt{\frac{b_{k}}{L}}
$$

where

$$
b_{k}=\frac{m^{3} g^{3}}{4\left(k^{2}+m^{2} g^{2} / 4\right)^{2}} .
$$

The interaction between collective bosons to this order is likewise independent of $K$ and is given by [5]

$$
f(q, \Delta, K)=\frac{8 m^{4} g^{5}}{L} \frac{q^{2}+\Delta^{2}-q \Delta+12 m^{2} g^{2}}{\left[(q-\Delta)^{2}+4 m^{2} g^{2}\right]\left(q^{2}+4 m^{2} g^{2}\right)\left(\Delta^{2}+4 m^{2} g^{2}\right)} .
$$

The above interaction is clearly repulsive, reflecting the fact that it originates solely from quark exchange. Furthermore, the repulsion is maximal for bosons with the same momentum, i.e., $\Delta=q=0$. As such, a condensate of $K=0$ bosons, though certainly lowest in kinetic energy, will not be the optimum means of exploiting the interaction. A natural way to optimally treat the short-range Pauli-based correlations that arise from this residual interaction is through the use of Brueckner theory, to which we turn in the following section.

\section{BRUECKNER THEORY}

\section{A. $G$-matrix treatment}

In order to take into account Brueckner correlations for a boson system governed by a Hamiltonian $H_{B}=H_{0}+V_{B}$, we introduce the $G$-matrix operator equation

$$
G=V_{B}+V_{B} \frac{Q}{\mathcal{E}_{0}-H_{0}} G,
$$

where $\mathcal{E}_{0}$ is the lowest $(K=0)$ eigenvalue of the unperturbed Hamiltonian $H_{0}$,

$$
H_{0}\left|\Phi_{0}\right\rangle=\mathcal{E}_{0}\left|\Phi_{0}\right\rangle, \quad\left\langle\Psi_{0}\right| H_{0}=\left\langle\Psi_{0}\right| \mathcal{E}_{0},
$$

and $Q$ is the projection operator out of the uncorrelated ground state $\left|\Phi_{0}\right\rangle$ or $\left\langle\Psi_{0}\right|$. The $G$-matrix operator connects the uncorrelated states $\left|\Phi_{0}\right\rangle$ and $\left\langle\Psi_{0}\right|$ with the correlated ones $|\Phi\rangle$ and $\langle\Psi|$, respectively, according to

$$
V_{B}|\Phi\rangle=G\left|\Phi_{0}\right\rangle, \quad\langle\Psi| V_{B}=\left\langle\Psi_{0}\right| G .
$$

Due to the non-Hermiticity of the interaction, $V_{B}^{\dagger} \neq V_{B}$, we are obviously dealing with a nonunitary $G$-matrix theory.

Using the complete set of eigenstates of $H_{0}$ and projecting, we obtain the matrix form of Eq. (36),

$$
\begin{aligned}
\left\langle\Psi_{K}|G| \Phi_{0}\right\rangle= & \left\langle\Psi_{K}\left|V_{B}\right| \Phi_{0}\right\rangle \\
& +\sum_{P \neq 0} \frac{\left\langle\Psi_{K}\left|V_{B}\right| \Phi_{P}\right\rangle\left\langle\Psi_{P}|G| \Phi_{0}\right\rangle}{\mathcal{E}_{0}-\mathcal{E}_{P}},
\end{aligned}
$$




$$
\begin{aligned}
\left\langle\Psi_{0}|G| \Phi_{K}\right\rangle= & \left\langle\Psi_{0}\left|V_{B}\right| \Phi_{K}\right\rangle \\
& +\sum_{P \neq 0} \frac{\left\langle\Psi_{P}\left|V_{B}\right| \Phi_{K}\right\rangle\left\langle\Psi_{0}|G| \Phi_{P}\right\rangle}{\mathcal{E}_{0}-\mathcal{E}_{P}} .
\end{aligned}
$$

The fact that we need two equations to fully specify the matrix elements of $G$ is again a reflection of the nonHermiticity of the Hamiltonian. Given the solution to these two equations, the total energy of the system is then obtained as

$$
E_{G}=\left\langle\Psi|H| \Phi_{0}\right\rangle=\left\langle\Psi_{0}|H| \Phi\right\rangle=\mathcal{E}_{0}+\left\langle\Psi_{0}|G| \Phi_{0}\right\rangle,
$$

where the correlated states are assumed to be normalized in the usual way according to

$$
\left\langle\Psi_{0} \mid \Phi\right\rangle=\left\langle\Psi \mid \Phi_{0}\right\rangle=1 .
$$

\section{B. Two-body correlations}

We restrict our consideration here only to two-body correlations among the particles, adopting for the problem the following set of states involving two noncondensed (i.e., $K$ $\neq 0$ ) bosons:

$$
\begin{aligned}
\left|\Phi_{K}\right\rangle= & \frac{1}{\sqrt{\left(N_{B}-2\right) !}} \Lambda_{K}^{\dagger} \Lambda_{-K}^{\dagger} \Lambda_{0}^{\dagger\left(N_{B}-2\right)}|0\rangle, \\
& \left\langle\Psi_{K}\right|=\frac{1}{\sqrt{\left(N_{B}-2\right) !}}\langle 0| \lambda_{0}{ }^{\left(N_{B}-2\right)} \lambda_{-K} \lambda_{K} .
\end{aligned}
$$

This set of states, together with the condensate states (22), form the two-body sector of the complete Fock space.

Not all of the terms that enter the complete collective boson Hamiltonian (19)-(21) contribute in the two-body sector. For those that do contribute, it is useful to split them according to

$$
H_{B} \rightarrow H_{0}^{\prime}+V_{B}^{\prime},
$$

where

$$
\begin{aligned}
H_{0}^{\prime}= & \epsilon_{0} \Lambda_{0}^{\dagger} \lambda_{0}+\sum_{K \neq 0} \epsilon_{K} \Lambda_{K}^{\dagger} \lambda_{K}+f(0,0,0) \Lambda_{0}^{\dagger} \Lambda_{0}^{\dagger} \lambda_{0} \lambda_{0} \\
& +4 \sum_{K \neq 0} f(K, K, K) \Lambda_{0}^{\dagger} \Lambda_{K}^{\dagger} \lambda_{K} \lambda_{0},
\end{aligned}
$$

and

$$
\begin{aligned}
V_{B}^{\prime}= & \sum_{K \neq 0} f(K, 2 K, K) \Lambda_{0}^{\dagger} \Lambda_{0}^{\dagger} \lambda_{K} \lambda_{-K} \\
& +\sum_{K \neq 0} f(K, 0, K) \Lambda_{K}^{\dagger} \Lambda_{-K}^{\dagger} \lambda_{0} \lambda_{0} \\
& +\sum_{K^{\prime}, K \neq 0} f\left(K-K^{\prime}, 2 K, K\right) \Lambda_{-K^{\prime}}^{\dagger} \Lambda_{K^{\prime}}^{\dagger} \lambda_{K^{\prime}} \lambda_{-K},
\end{aligned}
$$

with $\epsilon_{K}$ and $f(q, \Delta, K)$ defined by Eqs. (20) and (21), respectively. The separation (43)-(45) has the property that

$$
\left\langle\Psi_{0}\left|V_{B}^{\prime}\right| \Phi_{0}\right\rangle=0, \quad\left\langle\Psi_{0}\left|H_{0}^{\prime}\right| \Phi_{0}\right\rangle=\mathcal{E}_{0}
$$

which shows that $V_{B}^{\prime}$ is a true residual interaction, and that the uncorrelated energy $\mathcal{E}_{0}$ is precisely the expectation value of $H_{0}^{\prime}$ taken between the condensate wave functions (22).

Using this form of the Hamiltonian, we obtain for the unperturbed ground state energy

$$
\mathcal{E}_{0}=\left\langle\Psi_{0}\left|H_{0}\right| \Phi_{0}\right\rangle=N_{B} \varepsilon_{0}+N_{B}\left(N_{B}-1\right) f(0,0,0),
$$

and for the unperturbed energies corresponding to the states (42)

$$
\begin{aligned}
\mathcal{E}_{K}= & \left\langle\Psi_{K}\left|H_{0}\right| \Phi_{K}\right\rangle \\
= & \left(N_{B}-2\right) \varepsilon_{0}+2 \varepsilon_{K}+\left(N_{B}-2\right)\left(N_{B}-3\right) f(0,0,0) \\
& +4\left(N_{B}-2\right)[f(K, K, K)+f(0, K, K)] .
\end{aligned}
$$

For large values of $N_{B}$, the energy difference $\mathcal{E}_{0}-\mathcal{E}_{K}$ is given by

$$
\mathcal{E}_{0}-\mathcal{E}_{K}=-\frac{K^{2}}{2 m}+4 N_{B}[f(0,0,0)-f(0, K, K)-f(K, K, K)],
$$

and the required matrix elements of the interaction $(K$ $\neq 0, K^{\prime} \neq 0$ ) by

$$
\begin{gathered}
\left\langle\Psi_{0}\left|V_{B}^{\prime}\right| \Phi_{0}\right\rangle=0, \quad\left\langle\Psi_{K}\left|V_{B}^{\prime}\right| \Phi_{0}\right\rangle=2 N_{B} f(K, 0, K), \\
\left\langle\Psi_{0}\left|V_{B}^{\prime}\right| \Phi_{K}\right\rangle=2 N_{B} f(K, 2 K, K), \\
\left\langle\Psi_{K^{\prime}}\left|V_{B}^{\prime}\right| \Phi_{K}\right\rangle=4 f\left(K-K^{\prime}, 2 K, K\right) .
\end{gathered}
$$

With the aid of the matrix elements given above, the $G$-matrix equations (39) become

$$
\begin{aligned}
\left\langle\Psi_{K}|G| \Phi_{0}\right\rangle= & 2 N_{B} f(K, 0, K) \\
& +2 \sum_{P \neq 0} \frac{f(P-K, 2 P, K)\left\langle\Psi_{P}|G| \Phi_{0}\right\rangle}{\mathcal{E}_{0}-\mathcal{E}_{P}}, \\
\left\langle\Psi_{0}|G| \Phi_{K}\right\rangle & \\
= & 2 N_{B} f(K, 2 K, K) \\
& +2 \sum_{P \neq 0} \frac{f(K-P, 2 K, K)\left\langle\Psi_{0}|G| \Phi_{P}\right\rangle}{\mathcal{E}_{0}-\mathcal{E}_{P}},
\end{aligned}
$$

$$
\begin{aligned}
\left\langle\Psi_{0}|G| \Phi_{0}\right\rangle & =N_{B} \sum_{P \neq 0} \frac{f(P, 2 P, 0)\left\langle\Psi_{P}|G| \Phi_{0}\right\rangle}{\mathcal{E}_{0}-\mathcal{E}_{P}} \\
& =N_{B} \sum_{P \neq 0} \frac{f(P, 0,0)\left\langle\Psi_{0}|G| \Phi_{P}\right\rangle}{\mathcal{E}_{0}-\mathcal{E}_{P}} .
\end{aligned}
$$

The total Brueckner energy of the system $\left(E_{G}\right)$ is obtained from the resulting $G$-matrix elements according to

$$
E_{G}=\mathcal{E}_{0}+N_{B} \sum_{P \neq 0} \frac{f(P, 2 P, 0)\left\langle\Psi_{P}|G| \Phi_{0}\right\rangle}{\mathcal{E}_{0}-\mathcal{E}_{P}} .
$$




\section{Scaling procedure}

As noted earlier, the two-color delta model scales according to the dimensionless parameter $\widetilde{\rho}=\rho /(m g)$. This scaling property can be used to simplify the numerical analysis. To do so, we introduce the following scaled quantities:

$$
\begin{gathered}
G^{S}(K)=\frac{4}{m g^{2}}\left\langle\Psi_{m g K}|G| \Phi_{0}\right\rangle, \\
\widetilde{G}^{S}(K)=\frac{4}{m g^{2}}\left\langle\Psi_{0}|G| \Phi_{m g K}\right\rangle, \\
f^{S}(K, P)=\frac{4 L}{\pi g} f(m g P-m g K, 2 m g P, m g K), \\
\widetilde{f}^{S}(K, P)=\frac{4 L}{\pi g} f(m g K-m g P, 2 m g K, m g K), \\
\widetilde{X}_{k}^{K}=\sqrt{N} X_{m g k}^{m g K}, \widetilde{Y}_{k}^{K}=\sqrt{N} Y_{m g k}^{m g K} .
\end{gathered}
$$

Using this notation and going to the infinite-matter limit leads to the following set of scaled $G$-matrix equations:

$$
\begin{aligned}
& G^{S}(K)=\pi \tilde{\rho} f^{S}(K, 0)-\int \frac{f^{S}(K, P) G^{S}(P)}{P^{2}-\widetilde{\rho} A^{S}(P)} d P, \\
& \widetilde{G}^{S}(K)=\pi \widetilde{\rho} \widetilde{f}^{S}(K, 0)-\int \frac{f^{S}(K, P) \widetilde{G}^{S}(P)}{P^{2}-\widetilde{\rho} A^{S}(P)} d P,
\end{aligned}
$$

where

$$
\begin{aligned}
f^{S}(K, P) & =\widetilde{f}^{S}(P, K) \\
& =\frac{8 \widetilde{\Delta}_{P}}{(\pi \widetilde{\rho})^{2}} \int \widetilde{Y}_{k^{\prime}+(P+K) / 2}^{K} \widetilde{Y}_{k^{\prime}-(P-K) / 2}^{K} \widetilde{X}_{k^{\prime}}^{P} d k^{\prime},
\end{aligned}
$$

and

$$
\begin{aligned}
A^{S}(P)= & \frac{8 \pi}{(\pi \widetilde{\rho})^{2}} \int d k^{\prime}\left[\widetilde{\Delta} \widetilde{Y}_{k^{\prime}}^{0} \widetilde{Y}_{k^{\prime}}^{0} \widetilde{X}_{k^{\prime}}^{0}-\widetilde{\Delta}_{P} \widetilde{Y}_{k^{\prime}}^{0} \widetilde{Y}_{k^{\prime}+P / 2}^{P} \widetilde{X}_{k^{\prime}}^{0}\right. \\
& -\widetilde{\Delta} \widetilde{Y}_{k^{\prime}}^{P} \widetilde{Y}_{k^{\prime}+P / 2}^{0} \widetilde{X}_{k^{\prime}}^{P}+\frac{1}{2}\left(\widetilde{\Delta}_{P} \widetilde{Y}_{k^{\prime}}^{P}-\widetilde{\Delta} \widetilde{Y}_{k^{\prime}}^{0}\right) \\
& \left.+\frac{1}{2} k^{\prime 2}\left(\widetilde{X}_{k^{\prime}}^{0} \widetilde{Y}_{k^{\prime}}^{0}-\widetilde{X}_{k^{\prime}}^{P} \widetilde{Y}_{k^{\prime}}^{P}\right)\right]
\end{aligned}
$$

with $\widetilde{\Delta}=1 /(2 \pi) \int X_{k}^{0} d k$ and $\widetilde{\Delta}_{K}=1 /(2 \pi) \int X_{k}^{K} d k$. It should be emphasized that in the infinite-matter limit all integrations can be extended to include the point $P=0$.

The set of equations (55) and (56) can be solved numerically, given a set of collective structure amplitudes $\left(\widetilde{X}_{k}^{K}, \widetilde{Y}_{k}^{K}\right)$. The total energy per particle is then given by

$$
\frac{E_{G}}{N} \frac{1}{m g^{2}}=\mathcal{E}_{0}^{S}+\Delta E_{G}^{S},
$$

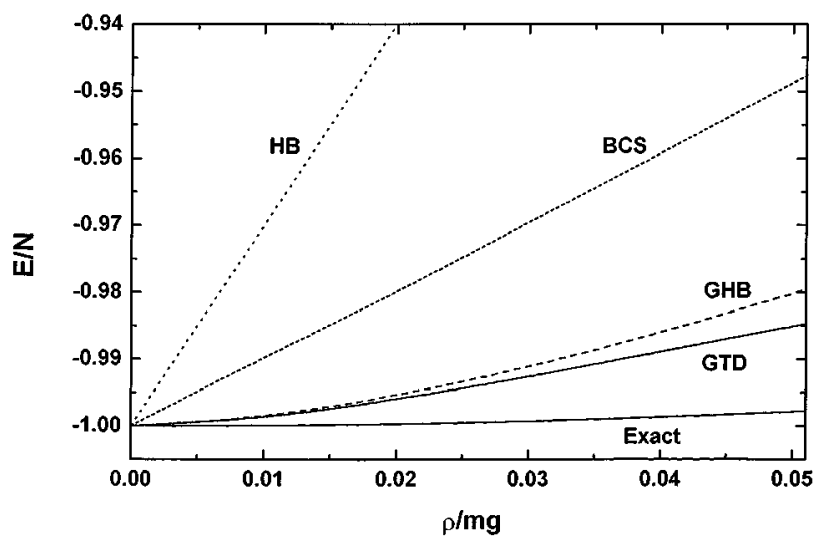

FIG. 1. The energy per quark (in units of $8 / \mathrm{mg}^{2}$ ) for the twocolor delta model as a function of the scaled density $\tilde{\rho}=\rho / \mathrm{mg}$. The various curves are described in the text.

where the first term is the uncorrelated ground state energy per particle

$$
\mathcal{E}_{0}^{S}=\frac{\mathcal{E}_{0}}{N} \frac{1}{m g^{2}}=\frac{1}{2 \pi \widetilde{\rho}} \int k^{2} X_{k}^{0} Y_{k}^{0} d k-\frac{\widetilde{\Delta}^{2}}{\widetilde{\rho}}
$$

and the second term represents the correlation energy

$$
\Delta E_{G}^{S}=\frac{\Delta E_{G}}{N} \frac{1}{m g^{2}}=-\frac{1}{2} \int \frac{f^{S}(0, P) G^{S}(P)}{P^{2}-\tilde{\rho} A^{S}(P)} d P .
$$

\section{Numerical results}

In Fig. 1, we plot the total energy per particle versus $\widetilde{\rho}$ for small densities, up to $\tilde{\rho}=0.05$. Two sets of Brueckner results are shown: (a) those denoted as GHB are obtained under the assumption that all collective bosons, independent of $K$, have the internal structure given in the HB approximation, (b) those denoted as GTD arise when the structure of the $K \neq 0$ bosons is taken from a boson TD analysis. These two sets of results are compared with the energies obtained using the exact Bethe ansatz (4), the BCS approximation (8), and the uncorrelated Hartree-Bose (HB) treatment (24). Several points can be readily seen from the figure.

(1) No linear contribution arises when Brueckner correlations are taken into account, in contrast to both the BCS and Hartree-Bose results. In fact, though fairly clear from the figure itself, this conclusion was actually reached by numerical differentiation of the energies at very low densities. Note further that this is the case for both the GHB and GTD prescriptions, reflecting the fact that the linear term in the energy is only sensitive to the zero-density structure amplitudes.

(2) The Brueckner energy is lower than both the BCS and Hartree-Bose energies, and in better agreement with the exact results.

(3) The use of $K$-dependent structure functions determined dynamically in TD approximation further improves the agreement with the exact results at these low densities. 


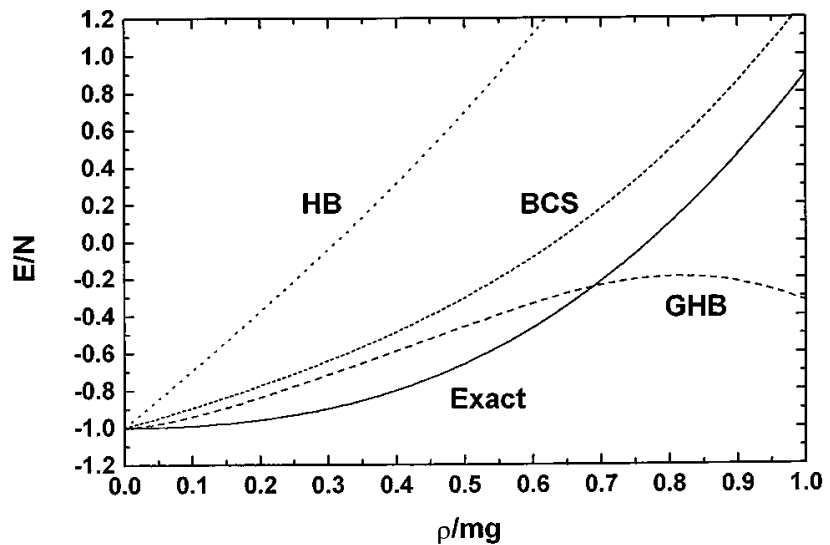

FIG. 2. The same as Fig. 1, but extended to $\tilde{p}=1$.

In Fig. 2, we extend the comparison to $\widetilde{\rho}=1$. We only show the Brueckner results under the simplifying assumption that all bosons have the same structure, as given by the HB approximation. Starting at a density of about $\widetilde{\rho} \approx 0.5$, the Brueckner results begin to show an unphysical behavior, and soon thereafter fall below the exact results. This can be readily understood. We have only included two-body Brueckner correlations in our analysis. It is well known, however, that as the density goes up, higher-body correlations play an increasingly more important role.

\section{DISCUSSION AND CONCLUDING REMARKS}

In this work, we have extended an earlier application of boson mapping techniques to a two-color delta model of interacting quarks. In an earlier work, the method of boson mappings was supplemented by a Hartree-Bose treatment of the resulting Hamiltonian, to obtain a variational description of the ground state of the system. Such a treatment gives rise to a linear term in the energy per quark as a function of density, a term that is not present in the exact results for that model. In the present analysis, we have improved on the Hartree-Bose treatment by including two-boson Brueckner correlations. To do this, we had to develop Brueckner theory for non-Hermitean Hamiltonians, which to the best of our knowledge has not been done before. The key result is that Brueckner correlations completely suppress the linear term in the density, in agreement with the exact results for the model, thereby leading to a much more accurate description of the underlying quark dynamics.

That Brueckner correlations might be important in describing the boson dynamics of this problem was already evident from the structure of the residual boson-boson interaction that derived from the Hartree-Bose analysis. This interaction is strongly repulsive at short distances, reflecting the effects of quark exchange when two composite bosons overlap. By incorporating Brueckner correlations, we are effectively suppressing those terms in the ground state wave function in which the bosons have the same momenta and thus overlap maximally.

There is an alternative, but equivalent, way of looking at the effect of Brueckner correlations. The fundamental difficulty in all approximate applications of mapping techniques, whether to bosons or baryons, is the admixture of unphysical states in the resulting wave functions. These unphysical admixtures represent Pauli-violating contributions to the wave function. The inclusion of Brueckner correlations can thus be viewed as a means of suppressing unphysical components in the ground states of composite-particle systems. The fact that our Brueckner analysis is able to reproduce the linear term in the density properly suggests that it is indeed a viable means of suppressing unphysical components following a mapping. Considering the great interest in developing procedures for removing unphysical (or spurious) components following any mapping [12], we believe that this is an important conclusion.

As emphasized in the Introduction, the analysis presented here is part of a larger program aimed at the application of baryon mapping techniques to semirealistic constituent quark models of real nuclei. Towards that end, it is important to reiterate the significance of these results to the more relevant three-color delta model studied in [6]. A baryon mapping of that model followed by a pure Hartree-Fock treatment likewise was unable to describe the exact dynamics at the lowest order in density in which the cluster-cluster interaction contributed. There, it was third order, however, due to the fermionic nature of baryons. The exact results gave a repulsive third-order contribution with coefficient $\pi^{2} / 729$; the baryon $\mathrm{HF}$ analysis gave a coefficient roughly six times larger. The fact that lowest-order Brueckner theory exactly corrects the first-order term in the energy of the two-color model suggests that it would likewise correct the corresponding thirdorder term of the three-color model.

Overall, our Brueckner + Hartree-Bose $(+$ TammDancoff) treatment of the two-color delta model provides the most accurate approximate description of its ground state dynamics at low densities to date. At higher densities, however, the agreement between our results and those obtained by exact solution of the model breaks down. We believe that this is a reflection of two limitations in the analysis.

(1) In our calculations, we have assumed that the structure of the dominant collective bosons can be taken from pure mean-field treatments prior to the inclusion of short-range Brueckner correlations. While this is probably true at fairly low densities, where the HB and/or the TD approximations should suffice in giving the dominant structure of the collective bosons, it does not seem to be the case at higher densities. There, we no doubt should be carrying out a fully selfconsistent Brueckner-Hartree-Bose treatment, in which the structure of the collective bosons is treated on the same footing as the short-range Brueckner correlations.

(2) We have only included in our Brueckner analysis twobody correlations. While this is appropriate at low densities, it is well known that at higher densities higher-body Brueckner correlations must be included.

Such extensions of the analysis, though numerically very demanding, are conceptually straightforward within the mapping framework described in this paper.

Were we to treat the three-color model analogously, disagreement with the exact results would no doubt show up at fourth-order in the density, for precisely the same reasons. Here too improvements are possible, by extending to a fully self-consistent Brueckner-Hartree-Fock treatment. However, the fact that these corrections would only affect the results at fourth order and higher suggests that they should not be especially important at the relatively low densities typical of realistic nuclei. As such, baryon mapping techniques coupled 
with a lowest-order Brueckner treatment of short-range correlation effects should be useful in treating quark cluster dynamics in realistic nuclear systems. Based on this conclusion, we are now planning to apply these methods to more realistic quark Hamiltonians [2].

It is useful to stress here another important difference between problems involving two-particle correlations (as in the two-color delta model) and three-particle correlations (as in the three-color delta model or more realistic quark models). When dealing with two-particle correlations, mapping techniques are useful, but not essential. Much the same physics can be incorporated in quasiparticle methods. When dealing with three-particle correlations, however, no analogous quasiparticle methods exist. As such, baryon mappings seem to provide a unique tool for treating such correlation effects. That being the case, it is of crucial importance that there now exists a method, namely non-Hermitean Brueckner theory, for suppressing unphysical components that result from quark exchange effects at short distances.

A limitation of the models discussed here is their restriction to nonrelativistic dynamics. This is probably acceptable for treating nuclear processes at low momentum transfers. However, it will no doubt be important to include relativistic effects at higher momentum transfers. While there is no conceptual difficulty in building relativity into the mapping formalism, this has not been worked out in detail yet.

Though our ultimate goal, as noted above, is the description of quarks in nuclei, the model that we have studied in this work is of importance to other areas of physics as well. In particular, it has generic relevance to boson mapping techniques and more specific relevance to superconducting properties of condensed matter systems [13].

\section{ACKNOWLEDGMENTS}

This work was supported in part by the National Science Foundation under Grant Nos. PHY-9303041 and INT9224875, by NATO under Grant No. CRG.900466, by the Bulgarian National Foundation for Scientific Research under Contract Nos. $\Phi-527$ and $\Phi-415$ and by the DGICYT (Spain) under Contract No. PB 92/0021-C02-01. One of the authors (M.V.S.) would like to acknowledge the support of a grant from the Fulbright Foreign Scholar Program. Useful discussions with Jonathan Engel in the early stages of this investigation are also gratefully acknowledged.
[1] M. Oka and K. Yazaki, Phys. Lett. 90B 41 (1980) ; K. Breuer, A. Faessler, F. Fernandez, and K. Shimizu, Nucl. Phys. A507 599 (1990).

[2] F. Fernandez, A. Valcarce, U. Straub, and A. Faessler, J. Phys. G 19, 2013 (1993).

[3] S. Pittel, J. Engel, J. Dukelsky, and P. Ring, Phys. Lett. B 247, 185 (1990).

[4] S. Pittel, J. M. Arias, J. Dukelsky, and A. Frank, Phys. Rev. C 50, 423 (1994).

[5] J. Dukelsky and S. Pittel, Phys. Rev. C 45, 1871 (1992).

[6] A. I. Georgieva, R. P. Roussev, P. P. Raychev, M. V. Stoitsov, S. Pittel, and J. Dukelsky, Phys. Rev. C 52, 2131 (1995).

[7] Abraham Klein and E. R. Marshalek, Rev. Mod. Phys. 63, 375 (1991).
[8] S. Tosa, Phys. Rev. C 34, 2302 (1986).

[9] D.S. Koltun and S. Tosa, Phys. Lett. B 172, 267 (1986); D.S. Koltun, Phys. Rev. C 36, 2047 (1987).

[10] P. Ring and P. Schuck, The Nuclear Many-Body Problem (Springer, Berlin, 1980); Amos deShalit and Herman Feshbach, Theoretical Nuclear Physics (John Wiley, New York, 1974), Vol. I.

[11] M. C. Cambiaggio and J. Dukelsky, Phys. Lett. B 197, 479 (1987).

[12] Da Hsuan Feng and Michel Vallieres, Int. J. Mod. Phys. E 283 (1993).

[13] J. Dukelsky, C. Esebbag, and M. de Llano, in Symmetries in Physics, edited by A. Frank and K. B. Wolf (Springer, Berlin, 1992) p. 35. 